\title{
Step line tension and step morphological evolution on the $\mathrm{Si}(111)(1 \times 1)$ surface
}

\author{
A. B. Pang, K. L. Man, and M. S. Altman* \\ Department of Physics, The Hong Kong University of Science and Technology, Clear Water Bay, Kowloon, Hong Kong \\ T. J. Stasevich, ${ }^{\dagger}$ F. Szalma, and T. L. Einstein \\ Department of Physics, University of Maryland, College Park, Maryland 20742-4111, USA \\ (Received 25 July 2007; revised manuscript received 6 December 2007; published 17 March 2008)
}

\begin{abstract}
The temperature dependence of the step line tension on the $\operatorname{Si}(111)(1 \times 1)$ surface is determined from a capillary wave analysis of two-dimensional island edge fluctuations and straight step fluctuations that are observed with low energy electron microscopy. The line tension decreases by nearly $20 \%$ with a linear temperature coefficient of $-0.14 \mathrm{meV} / \AA \mathrm{K}$ between 1145 and $1233 \mathrm{~K}$. Temporal correlations of step fluctuations exhibit the distinctive signature in the wavelength dependence of the relaxation time of a terrace diffusion-limited mechanism for step motion. We also find that the role of desorption in island decay increases dramatically in the temperature range $(1145-1380 \mathrm{~K})$ that island decay is studied. Consequently, we generalize the current quasistatic model of island decay to take account of desorption. The evaluation of the island decay time with this model referenced to the temperature-dependent line tension accurately determines activation energies that are relevant to mass transport and sublimation.
\end{abstract}

DOI: 10.1103/PhysRevB.77.115424

PACS number(s): 68.35.Md, 68.43.Jk, 68.37.Nq, 68.35.Fx

\section{INTRODUCTION}

In simple phenomenological models of crystalline surfaces, the step stiffness can play a prominent role in defining equilibrium step configurations and in governing step morphological evolution. The step stiffness is defined, $\widetilde{\beta}=\beta$ $+\partial^{2} \beta / \partial \theta^{2}$, in terms of the step line tension $\beta$, where $\theta$ is the azimuthal angle, and as such is a measure of the tendency of a step to remain straight. ${ }^{1}$ One important way in which the step stiffness may exert its influence on step morphology is through its presence in the Gibbs-Thomson (GT) relation, which figures generally in descriptions of curved surfaces. ${ }^{2}$ In the context of crystalline surfaces, the GT relation has frequently been used to express the dependence of the adatom concentration in equilibrium with an atomic step upon step curvature. ${ }^{3-14}$ This dependence plays an important role in several phenomena that affect surface morphology, such as the response of a step to shape perturbations ${ }^{8}$ including step flow instabilities ${ }^{9}$ and island coarsening and decay. ${ }^{3-7,10-12,15}$ Therefore, accurate knowledge of step stiffness or line tension, including their temperature dependence, should contribute to the understanding of many step morphological phenomena.

Steps on the $\operatorname{Si}(111)(1 \times 1)$ surface, which are the subject of the investigations described here, have been studied widely ${ }^{3,8,15-33}$ due to their intriguing phenomenology. The $\mathrm{Si}(111)$ surface undergoes a structural phase transition between $(7 \times 7)$ and $(1 \times 1)$ configurations at a transition temperature of $T_{c}=1133 \mathrm{~K}$. The step line tension is expected to be nearly isotropic on the $\mathrm{Si}(111)(1 \times 1)$ surface above $T_{c}{ }^{16}$ Under this condition, the step stiffness will be equal to and can be used interchangeably with the line tension. Several values of the step stiffness and line tension have been reported for the $\mathrm{Si}(111)(1 \times 1)$ surface at a few temperatures based on measurements that were made using reflection electron microscopy (REM). These values cover a fairly large range. Step stiffness was originally derived from measure- ments of the mean-square displacement of steps during equilibrium fluctuations. ${ }^{17}$ Stiffness values of 69 and $38 \mathrm{meV} / \AA$ were determined from the fluctuation behavior of two different steps at $1173 \mathrm{~K} .{ }^{17}$ These values were later revised upward by a factor of $2,{ }^{18}$ and finally a single lower value of $46 \mathrm{meV} / \AA$ was settled on after further corrections were made to the analysis. ${ }^{19}$ This is a little larger than the stiffness of $30 \mathrm{meV} / \AA$ that was determined earlier at $1173 \mathrm{~K}$ from an evaluation of the time correlation functions for the different Fourier modes of equilibrium step fluctuations. ${ }^{20}$ The stiffness was determined from the mean-square fluctuation displacement at $1323 \mathrm{~K}$ to be $3.2 \mathrm{meV} / \AA$. $^{21}$ The line tension was also reported to be $18.8 \mathrm{meV} / \AA$ at this temperature based on an evaluation of the equilibrium Si crystal shape. ${ }^{22}$ At $1373 \mathrm{~K}$, stiffness was determined to be $16.3 \pm 1.8 \mathrm{meV} / \AA$ from step diffusivity via measurement of the spatial correlation function..$^{23}$ A slightly smaller value of $12 \mathrm{meV} / \AA$ was determined at this temperature in the same work from the mean-square displacement due to fluctuations. ${ }^{23}$ The stiffness at $1373 \mathrm{~K}$ was later reported to be between 22.8 and $31.9 \mathrm{meV} / \AA .^{24}$ It was noted in that work that desorption is significant at $1373 \mathrm{~K}$. Therefore, care was taken to deposit a replenishing flux of $\mathrm{Si}$ atoms that compensated the desorption flux. ${ }^{23,24}$ It is not clear if the surface is in equilibrium or just in steady state under these conditions. On the other hand, values of a quantity called the dynamical step edge stiffness that were determined under dynamical conditions of sublimation between 1230 and $1380 \mathrm{~K}$ are orders of magnitude larger. ${ }^{8}$ If we disregard the dynamical stiffness for the moment and focus only on the most recent results obtained under equilibrium or steadystate conditions at $1173 \mathrm{~K}$ (Ref. 19) and $1373 \mathrm{~K}$ (Ref. 24), then the decrease of the step stiffness with increasing temperature is found to be in qualitative agreement with theoretical expectations. ${ }^{16}$ However, the variation of the reported values and the limited number of temperatures that were considered in separate experiments suggest that the issue of step 
stiffness or line tension on the $\mathrm{Si}(111)(1 \times 1)$ surface is not settled entirely.

In the present work, we have measured island edge fluctuations on the $\mathrm{Si}(111)(1 \times 1)$ surface in the temperature range of $1145-1233 \mathrm{~K}$ using low energy electron microscopy (LEEM). Line tension is determined by evaluating island edge fluctuations with an appropriate capillary wave method that was outlined recently in Ref. 34. The line tension that is determined from island edge fluctuations is then compared to the result of a more traditional analysis of straight step edge fluctuations that were observed with LEEM at $1163 \mathrm{~K}$. By consolidating results that are determined at different temperatures and for different geometries in one consistent set of measurements and analyses, we obtain a coherent view of the temperature-dependent line tension on the $\mathrm{Si}(111)(1 \times 1)$ surface. The importance of this result is highlighted here by measurements of island decay, which is driven by the GT effect. The role of desorption in island decay varies from negligible to dominant in the temperature range $(1145-1380 \mathrm{~K})$ that island decay is studied here. Therefore, the current model of island decay that neglects desorption is generalized to account for the increasing importance of desorption at higher temperature. The evaluation of the temperature-dependent island decay time with this general model, referenced to the temperature-dependent line tension, accurately determines activation energies that are relevant to mass transport and sublimation.

\section{MODEL OF ISLAND DECAY INCLUSIVE OF DESORPTION}

The thermodynamic driving force for island decay is the chemical potential difference between the island edge and its surroundings. The excess chemical potential of a step at an island edge is given by ${ }^{1,4,5,10,14,35}$

$$
\mu(r)=\Omega \widetilde{\beta} K(r),
$$

where $\Omega$ is the area per atom and $K(r)$ is the step curvature. For circular islands with radius $r, \widetilde{\beta}=\beta$ and $K(r)=1 / r$. The adatom concentration in equilibrium with a step is defined by the chemical potential through the GT relation ${ }^{1-7,12,14}$

$$
n_{e q}(r)=n_{e q}(\infty) \exp \left(\frac{\mu(r)}{k T}\right),
$$

where $n_{e q}(\infty)=\Omega^{-1} \exp \left(-E_{a d} / k T\right)$ is the concentration in equilibrium with a straight step, $k$ is the Boltzmann constant, $T$ is the temperature, and $E_{a d}$ is the adatom formation energy by detachment from a step. It is important to recognize that these expressions are appropriate for a material with one atom per unit cell. Since the $\mathrm{Si}(111)(1 \times 1)$ surface has two atoms per unit cell due to its bilayer structure, it is reasonable to expect that the detachment of a single atom from a step edge on $\mathrm{Si}(111)$ would leave its partner atom in the unit cell in a highly metastable state. Thus, detachment could be viewed as a process that involves both atoms in a unit cell, quasisimultaneously producing two adatoms on the terrace. This would require modification of the straight step equilibrium concentration to a form $n_{e q}(\infty)=2 \Omega^{-1} \exp \left(-E_{a d} / k T\right)$, where $E_{a d}$ is understood to be the formation energy per adatom pair and $\Omega=\sqrt{3} a^{2} / 2$ is the $\operatorname{Si}(111)$ surface unit cell area expressed in terms of the surface lattice constant, $a$ $=3.84 \AA$. With the view that step motion is mediated by the attachment and detachment of atom pairs, the area in Eq. (1) is also understood to be the unit cell area, $\Omega$.

According to current models of island decay, ${ }^{4-6,12}$ which neglect desorption, island area decreases via the serial detachment of atoms from the island edge, adatom diffusion across a terrace, and reattachment to a nearby step with lower chemical potential. However, the onset of desorption at high temperature presents an additional pathway for atoms to follow when they disperse from an island after detachment. Therefore, the current models are not expected to properly describe island decay under these conditions. In order to address this deficiency, we develop a more general model of island decay here that takes account of desorption.

We consider the decay of an "inner" circular island of radius $r_{i}$ that resides on a larger "outer" circular island of radius $r_{o}$. The current densities of atoms that detach from and attach to the perimeter of the inner island are

$$
\begin{aligned}
& j_{d e t}=\kappa n_{e q}\left(r_{i}\right), \\
& j_{a t t}=-\kappa n\left(r_{i}\right),
\end{aligned}
$$

where $\kappa$ is the kinetic coefficient for attachment/detachment and $n\left(r_{i}\right)$ is the actual concentration at the island perimeter. Thus, the net adatom current density at the perimeter is

$$
j_{n e t}=\kappa\left[n_{e q}\left(r_{i}\right)-n\left(r_{i}\right)\right],
$$

and the rate at which the island area $A$ changes is

$$
\frac{d A}{d t}=-\Omega j_{n e t} 2 \pi r_{i}
$$

Since Eq. (5) is written in terms of the adatom current density $j_{\text {net }}$, the area in this expression must refer to the atomic area in the island. In the case of the bilayer step on the $\mathrm{Si}(111)(1 \times 1)$ surface, each detaching or attaching atom takes away or adds one-half of the unit cell area, $\Omega / 2$. Equation (5) should be modified accordingly.

The adatom concentration obeys the stationary diffusion equation between the step boundaries at the edges of the inner and outer islands,

$$
D \nabla^{2} n-\frac{n}{\tau}=0
$$

where $D=\left(n_{s} / 4\right) a^{2} \nu_{0} \exp \left(-E_{d i f} / k T\right)$ is the diffusion constant, $n_{s}$ is the number of nearest neighbor sites $\left(n_{s}=3,4\right.$, and 6 for 
honeycomb, square, and triangular lattices, respectively), ${ }^{10,36}$ $\tau=\nu_{0}^{-1} \exp \left(E_{\text {des }} / k T\right)$ is the adatom lifetime prior to desorption, $\nu_{0}$ is the attempt frequency which is assumed to be the same for desorption and diffusion, $E_{d i f}$ is the diffusion energy, and $E_{d e s}$ is the desorption energy. The key difference from the earlier models of island decay is the inclusion in Eq. (6) of the desorption term.

The general solution of the two-dimensional diffusion equation in circular coordinates is

$$
n(r)=B_{I} I_{0}(\widetilde{r})+B_{K} K_{0}(\widetilde{r}),
$$

where $I_{p}$ and $K_{p}$ are the $p$ th-order [ $p=0$ in Eq. (7)] modified Bessel functions of the first and second kinds, respectively. We use reduced spatial coordinates $\tilde{r}=r / x_{s}$, where $x_{s}=\sqrt{D \tau}$ $=\left(\sqrt{n_{s}} / 2\right) a \exp \left[\left(E_{d e s}-E_{d i f}\right) / 2 k T\right]$ is the diffusion length prior to desorption. Explicit expressions for the coefficients, $B_{I}$ and $B_{K}$, are found by imposing the following boundary conditions at the inner and outer boundaries:

$$
\begin{aligned}
& -\left.D \frac{d n}{d r}\right|_{r_{i}}=\kappa\left[n_{e q}\left(r_{i}\right)-n\left(r_{i}\right)\right], \\
& -\left.D \frac{d n}{d r}\right|_{r_{o}}=\kappa\left[n\left(r_{o}\right)-n_{e q}\left(r_{o}\right)\right] .
\end{aligned}
$$

These boundary conditions state that the net current densities of atoms that detach from the inner island perimeter (righthand side of (a)) and attach to the outer island perimeter (right-hand side of (b)) are equal to the adatom current densities on the terrace at these points (left-hand sides). In Eqs. (8a) and (8b), we have implicitly taken the boundaries to be impermeable. This is justified in the present study of the $\operatorname{Si}(111)(1 \times 1)$ surface because the recent observation of mass conservation during the decay of island stacks on this surface is a strong indication of impermeability. ${ }^{33}$ We have also assumed that the kinetic coefficients $\kappa$ at the inner and outer boundaries are equal. Kinetic coefficient asymmetry can be easily incorporated in the model at this point, if desired. Note that the actual adatom concentration is sometimes approximated to be equal to the equilibrium concentration at the outer boundary, $n\left(r_{o}\right) \approx n_{e q}\left(r_{o}\right)^{4,6}$ While this may be a good approximation when the outer island radius is large compared to the inner island radius or possibly also in the diffusion-limited regime, Eq. (8b) is a more accurate treatment of the boundary condition. The coefficients are then determined to be

$$
\begin{gathered}
B_{I}=\frac{C_{K} n_{e q}\left(r_{i}\right)+D_{K} n_{e q}\left(r_{o}\right)}{D_{K} C_{I}+C_{K} D_{I}}, \\
B_{K}=\frac{-C_{I} n_{e q}\left(r_{i}\right)+D_{I} n_{e q}\left(r_{o}\right)}{D_{K} C_{I}+C_{K} D_{I}},
\end{gathered}
$$

where

$$
\begin{gathered}
C_{K}=K_{0}\left(\widetilde{r}_{o}\right)-\tilde{d} K_{1}\left(\widetilde{r}_{o}\right), \quad D_{K}=-K_{0}\left(\widetilde{r}_{i}\right)-\tilde{d} K_{1}\left(\widetilde{r}_{i}\right), \\
C_{I}=I_{0}\left(\widetilde{r}_{o}\right)+\tilde{d} I_{1}\left(\widetilde{r}_{o}\right), \quad D_{I}=I_{0}\left(\widetilde{r}_{i}\right)-\tilde{d} I_{1}\left(\widetilde{r}_{i}\right),
\end{gathered}
$$

where $\tilde{d}=d / x_{s}$ is dimensionless and $d=D / \kappa$ is the kinetic length, which characterizes the rate-limiting step continuously between the diffusion limited $(d=0)$ and the attachment-detachment limited $(d=\infty)$ extremes. Note that the solution for the approximate outer boundary condition, $n\left(r_{o}\right) \approx n_{e q}\left(r_{o}\right)$, is obtained by setting $C_{K}=K_{0}\left(\widetilde{r}_{o}\right)$ and $C_{I}=I_{0}\left(\widetilde{r}_{o}\right)$.

After evaluating $n\left(r_{i}\right)$ using Eqs. (7), (9a), and (9b) and substituting the result into Eq. (4) to determine $j_{\text {net }}$, the rate of change of the island area is found by Eq. (5) to be

$$
\frac{d A}{d t}=-2 \pi \widetilde{r}_{i} \frac{n_{s}}{4} a^{2} \nu_{0} \exp \left(-\frac{E_{a d}+E_{d i f}}{k T}\right) \frac{\left[D_{I} K_{1}\left(\widetilde{r}_{i}\right)-D_{K} I_{1}\left(\widetilde{r}_{i}\right)\right] \exp \left(\frac{\xi}{r_{o}}\right)-\left[C_{K} I_{1}\left(\widetilde{r}_{i}\right)+C_{I} K_{1}\left(\widetilde{r}_{i}\right)\right] \exp \left(\frac{\xi}{r_{i}}\right)}{C_{I} D_{K}+C_{K} D_{I}} .
$$

In Eq. (10), the characteristic length is defined as $\xi$ $\equiv \widetilde{\beta} \Omega / k T \cdot{ }^{14,30}$ Note that this equation is also valid after taking account of the $\mathrm{Si}(111)$ bilayer step structure. Equation (10) indicates that the island decay rate depends explicitly on the sum of activation energies $E_{1}=E_{a d}+E_{d i f}$, in agreement with earlier models. ${ }^{4,5}$ The decay rate is also predicted to be sensitive to the difference $E_{2}=E_{d e s}-E_{d i f}$ through the dependence of Eq. (10) on the diffusion length. In the limit that the diffusion length approaches infinity, i.e., $E_{2}$ is large and desorption is negligible, the decay rate given by Eq. (10) simplifies to

$$
\begin{aligned}
\lim _{x_{s} \rightarrow \infty} \frac{d A}{d t}= & -2 \pi \frac{n_{s}}{4} a^{2} \nu_{0} \\
& \times \exp \left(-\frac{E_{a d}+E_{d i f}}{k T}\right) \frac{\exp \left(\frac{\xi}{r_{i}}\right)-\exp \left(\frac{\xi}{r_{o}}\right)}{\ln \left(\frac{r_{o}}{r_{i}}\right)+\frac{d}{r_{i}}+\frac{d}{r_{o}}} .
\end{aligned}
$$

The limiting solution for the approximate boundary condi- 
tion, $n\left(r_{o}\right) \approx n_{e q}\left(r_{o}\right)$, is then obtained by setting the last term in the denominator of Eq. (11) to zero $(d=0$ or $\kappa=\infty$ at the outer boundary). The resulting expression reproduces the solution that was derived earlier for this approximate boundary condition in the absence of desorption. ${ }^{4}$

If desorption is not negligible, then one must also consider the effect of desorption from the top of the island. Desorption will reduce the adatom concentration below the equilibrium value on the top of the island. This will result in the inward detachment of atoms from the island step edge, which will consequently increase the decay rate. If we treat the inward and outward detachments of atoms as independent channels, then an analogous expression for the island decay rate due only to desorption from the top of the island can be derived following the procedure given above. The boundary conditions on the top of the island are

$$
-\left.D \frac{d n}{d r}\right|_{r_{i}}=\kappa\left[n\left(r_{i}\right)-n_{e q}\left(r_{i}\right)\right]
$$

and that the concentration at the center of the island, $r=0$, remain finite. These lead to the coefficients in the general solution [Eq. (7)] of

$$
B_{I}=\frac{n_{e q}\left(r_{i}\right)}{I_{0}\left(\widetilde{r}_{i}\right)+\widetilde{d} I_{1}\left(\widetilde{r}_{i}\right)} .
$$

and $B_{K}=0$. The corresponding contribution of desorption from the top of the island to the decay rate is found by similar methods to be

$$
\left(\frac{d A}{d t}\right)_{\text {top }}=-2 \pi \widetilde{r}_{i} \frac{n_{s}}{4} a^{2} \nu_{0} \exp \left(-\frac{E_{a d}+E_{d i f}}{k T}\right) \frac{I_{1}\left(\widetilde{r}_{i}\right) \exp \left(\frac{\xi}{r_{i}}\right)}{I_{0}\left(\widetilde{r}_{i}\right)+\widetilde{d} I_{1}\left(\widetilde{r}_{i}\right)} .
$$

The total decay rate is then the sum of Eqs. (10) and (12).

\section{EXPERIMENTAL DETAILS}

The Si samples that were used in the experiments were miscut by $0.1^{\circ}$ from the (111) direction. Doping was $n$ type (phosphorous) with resistivity $10 \Omega \mathrm{cm}$. Island decay was observed on the tops of circular mounds that were fabricated by a photolithographic method. ${ }^{15}$ The sample was heated by electron bombardment from the rear. Sample temperature measurements were performed with an optical pyrometer and a W-3\% Re/W-25\% Re thermocouple spot welded to the sample holder immediately adjacent to the sample. The emissivity setting of the pyrometer was calibrated at $T_{c}$ $=1133 \mathrm{~K}$ defined by LEEM observations of continuous step decoration by the $(7 \times 7)$ structure. This determined an emissivity setting of 0.46 , which is comparable to values that were reported for $\mathrm{Si}$ with similar doping. ${ }^{37}$ Temperature measurement relative to $T_{c}$ was then accurate to within $3 \mathrm{~K}$.

The experiments were carried out using a LEEM with base pressure of $5 \times 10^{-11}$ torr. The imaging principle and real-time capability of LEEM have been described previously. ${ }^{38}$ LEEM step contrast ${ }^{39}$ is exploited here to ob- serve island decay. An imaging electron energy of $10 \mathrm{eV}$ was used for imaging. Although the interference condition is nearly optimal for step contrast at this energy, step contrast is still rather weak and subject to the detrimental effect of image noise. The noise level was reduced by integrating images for $0.55 \mathrm{~s}$, which resulted in an image acquisition rate of just under 2 frames/s. Images were digitized with a pixel density that corresponded to a pixel resolution of $6.8 \mathrm{~nm}$. This matches roughly the theoretical electron-optically defined instrumental resolution.

Due to the weak step contrast, it was necessary to perform spatial averaging in addition to the temporal image averaging in order to determine step configurations accurately. For the island geometry, radial line scans were first made through steps at island edges with polar angle increments that corresponded to pixel resolution along the island perimeter. Each radial line profile was then averaged with nine neighboring profiles on either side. For the straight step geometry, line profiles perpendicular to the step were measured at each point along the step with pixel resolution. Each line profile was then averaged with nine similar neighboring line profiles on either side. Although this spatial averaging corrupts the measurement of step fine structures having lengths comparable to or shorter than the averaging length scale $(130 \mathrm{~nm})$, it helps in the measurement of step coarse structures that exceed the averaging length scale sufficiently. The analyses of step line tension and step fluctuation temporal correlations, which are described in Sec. IV, focus on long wavelength fluctuation modes that are accessible to the measurement both spatially and temporally.

\section{EXPERIMENTAL RESULTS}

\section{A. Step line tension}

We determine the step line tension from a capillary wave analysis of equilibrium step fluctuations. Fluctuations of steps at the edges of two-dimensional islands were measured during island decay in the temperature range of $1145-1233 \mathrm{~K}$. At these temperatures, island decay is slow. Therefore, fluctuations may be considered to be close to equilibrium. These investigations are supplemented by similar investigations of straight step fluctuations at a single temperature $(1163 \mathrm{~K})$ within the temperature range of the island edge fluctuation measurements.

The analysis of step fluctuations typically begins by defining different fluctuation modes through a Fourier transform of the step configuration at time $t$. For straight steps, this has the form ${ }^{20,23,40-43}$

$$
x(y, t)=\sum_{q} x_{q}(t) \exp (\text { iqy }),
$$

where $x$ is the perpendicular displacement of the step from the mean step position, $y$ is the position along the step, $x_{q}(t)$ is the Fourier amplitude for mode $q=2 \pi / \lambda$, and $\lambda$ is the fluctuation wavelength. The possible wavelengths are $\lambda_{m}$ $=L / \mathrm{m}$, where $L$ is the length of the step that is being analyzed and $m=1,2,3, \ldots, m_{\max }$. We interchangeably label the modes with $q$ and $m$ in the following discussion. An analo- 


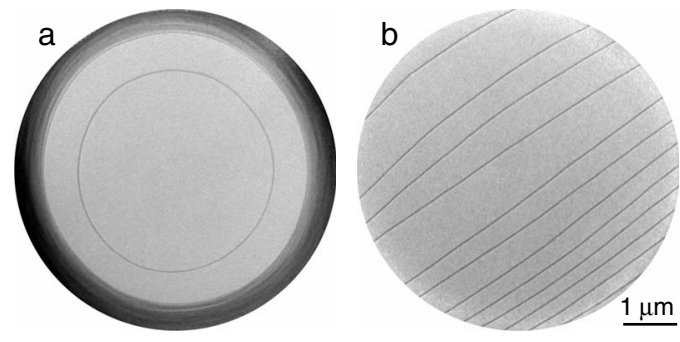

FIG. 1. LEEM images of (a) monoatomic height islands on prefabricated mounds and (b) straight monoatomic height steps on the $\mathrm{Si}(111)(1 \times 1)$ surface at $1163 \mathrm{~K}$ are shown. The imaging energy was $10 \mathrm{eV}$.

gous expression can be written in polar coordinates for island edges by replacing $x$ with $r, x_{q}(t)$ with $r_{q}(t)$, and $y$ with $R \theta$, which represents a position on the time-averaged island shape with mean radius $R$. The step length in this case is equal to the island perimeter.

In order to determine the line tension, we must first select the appropriate "window" in the fluctuation spectrum. The limits of the window are defined by the spatial and temporal characteristics of the measurement. The longest $(m=1)$ and shortest $\left(m=m_{\max }\right)$ possible wavelengths that we may consider are determined by the step length $L$ that is being analyzed and by the shortest experimentally discernible length, i.e., the pixel size, respectively. Each mode also has its own natural relaxation time, as we will discuss in the next section. We discard those long wavelength modes whose relaxation times are longer than about $\sim 5 \%$ of the total measurement time (typically a few hundred seconds in our experiments) because they do not move through enough fluctuation cycles during the measurement to provide statistically significant results. At the other end of the spectrum, short wavelength modes whose relaxation times are shorter than the image integration time $(0.55 \mathrm{~s}$ here $)$ are disregarded because of temporal averaging of the step position during image acquisition. They are also obscured by noise. We only consider modes in the window between these two temporal limits.

One advantage of focusing on island edge fluctuations in this work is that the perimeter of an island can be longer than a straight step that spans the same field of view. This imparts higher resolution of the fluctuation modes, $q$. Consequently, more modes are available for analysis in the appropriate spectral window. Islands also have well defined step lengths, given by their perimeters, while in the case of straight steps one must also consider the problem of the effective step length. ${ }^{34,44}$ On the other hand, island decay brings the practical disadvantage that the island radius decreases continuously during the fluctuation measurement due to decay. This limits the number of consecutive step configurations that can be recorded in an image sequence at approximately the same island radius, and therefore also the total measurement time. Consequently, information that is obtained from the analysis of fluctuation modes at the long wavelength end of the spectrum, i.e., long relaxation time, may be rendered less reliable or subject to greater uncertainty. This disadvantage is mitigated in our work by evaluating fluctuations of numerous islands over short periods, typically corresponding to about a

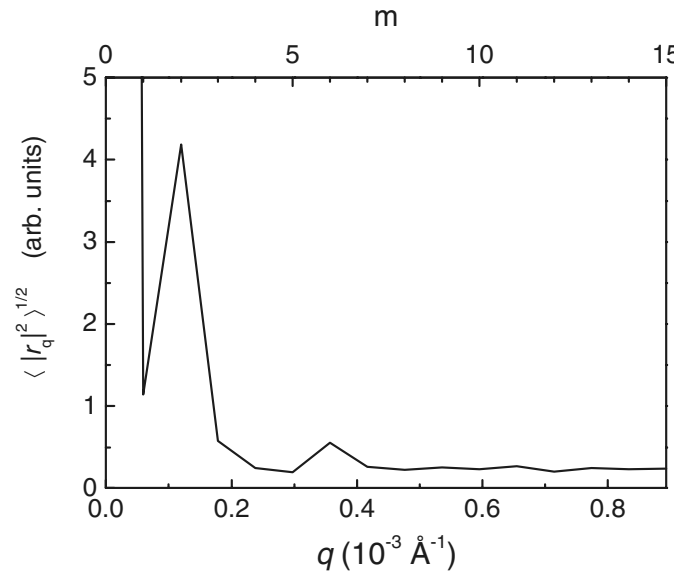

FIG. 2. The Fourier mode amplitudes of the time-averaged island shape at $T=1163 \mathrm{~K}$ indicates that the anisotropy is small. The $m=3$ and $m=6$ components identify anisotropy that is related to the surface symmetry.

$10 \%$ reduction of the island radius, and then averaging the results. This approach is facilitated by observing islands on the tops of prefabricated mounds, which serve as reproducible island sources and platforms for decay. An example is shown in Fig. 1(a). In this way, nearly identical island configurations are reproduced repeatedly. An example of the straight step configuration that was investigated is shown in Fig. 1(b).

Figure 2 presents the Fourier mode amplitudes of the time-averaged island shape. In this figure, the $m=0$ mode is the island radius, $m=1$ is due to an offset of the island position from the origin, and $m=2$ is an elliptical island shape distortion that is somehow imposed by the global mound shape, which was slightly elliptical. The small peaks in Fig. 2 for the $m=3$ and $m=6$ modes are expected for the threefold surface symmetry. The $m=2$ Fourier amplitude shown in Fig. 2 is much more sensitive to ellipticity than visual inspection of images. This serves to emphasize that the $m=3$ and $m$ $=6$ components are really very small, amounting to an island shape anisotropy that is less than $1 \%$ at $1163 \mathrm{~K}$. Therefore, it is a good approximation to treat islands on the $\mathrm{Si}(111)$ (1 $\times 1)$ surface at elevated temperatures as circular in shape.

We use a simplified version of the island edge fluctuation analysis described in Ref. 34 for the case of a perfect circular symmetry that is approximated well by the experiment. In particular, the stiffness, which is equal to the isotropic line tension, is related to the fluctuation amplitude for each mode according to

$$
\beta=\frac{k T}{2 \pi R q^{2}\left\langle\left|r_{q}(t)\right|^{2}\right\rangle},
$$

where the average of the Fourier amplitude is both a time and ensemble average. We examined islands with mean radius during a slow decay of $R \sim 1.7 \mu \mathrm{m}$ at several temperatures. Figure 3 shows the line tension values that are determined by Eq. (13) at $1163 \mathrm{~K}$ for many modes. Each Fourier mode should lead to the same line tension based on the equipartition theorem, provided that the mode is not adversely 

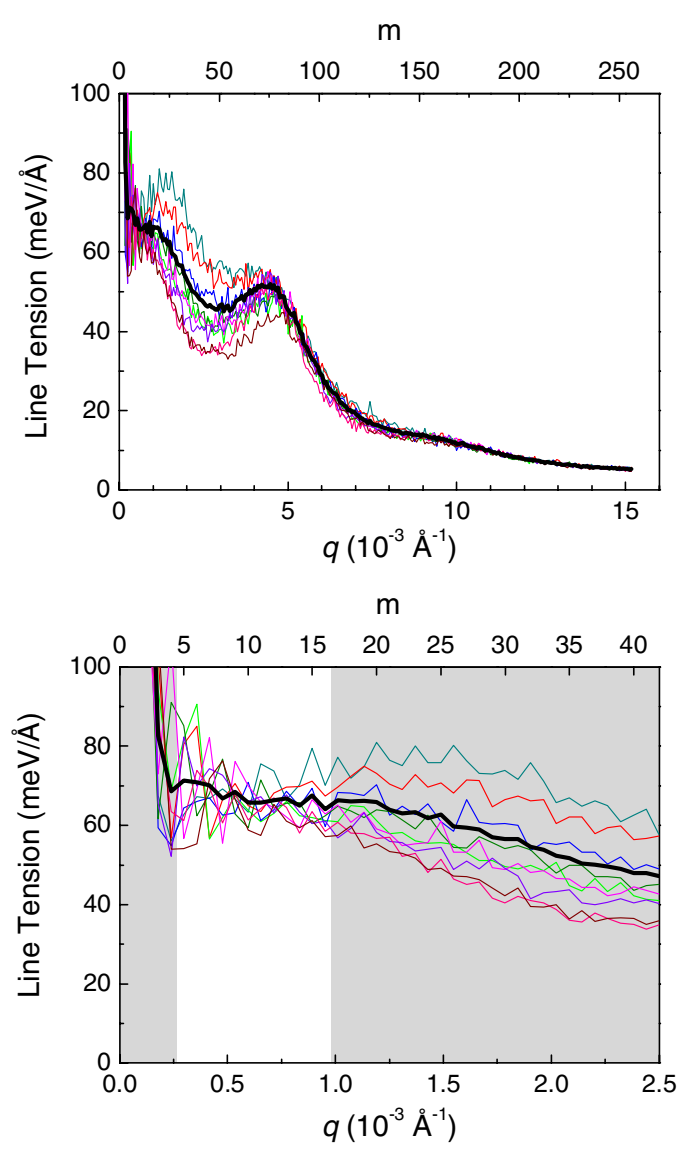

FIG. 3. (Color online) Step line tension vs fluctuation mode determined from an analysis of island fluctuations at $1163 \mathrm{~K}$. Each thin curve was obtained from an independent data set. The thicker black curve is the average of the individual results. In (a), the anomalous supression of results caused by noise at short wavelength and a spurious peak (at $m \sim 75$ ) caused by spatial averaging are observed. In (b), step line tension is shown in greater detail for long wavelength modes of interest. The step line tension was determined by the average of the values in the unshaded window, 5 $\leqslant m \leqslant 16$, in (b).

affected by the temporal limitations that are discussed above. We identify the modes that fall in this spectral window by considering the mode-dependent relaxation times that are obtained from evaluating the temporal correlation function. The correlation function analysis is described in Sec. IV B. At $1163 \mathrm{~K}$, we find that the 16 longest wavelength modes have relaxation times that are longer than the image integration time. The $m=5$ mode is chosen as the long wavelength cutoff. This mode has a relaxation time that is shorter than $1 / 30$ of the total measurement time at $1163 \mathrm{~K}, 9.2 \mathrm{~min}$, which corresponds to a sequence of 1000 images. The line tension is determined by averaging the values for modes in the window $5 \leqslant m \leqslant 16$.

It is worth noting that the line tension apparently decreases in Fig. 3 for short wavelength modes, $m>20$, and that a peak is present at mode $m \sim 75$. The anomalous decrease is due to the greater importance of noise at short wavelengths. The peak is caused by the spatial averaging that we perform in order to reduce noise and determine step position accurately. This procedure is described in Sec. III. The

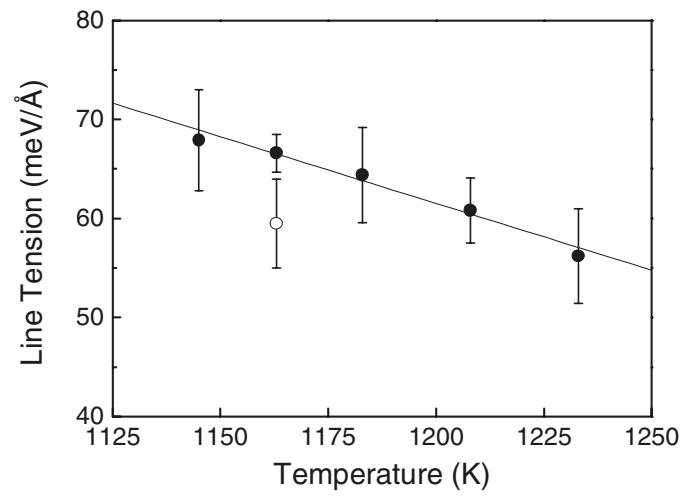

FIG. 4. The step line tension determined from island fluctuations $(\bigcirc)$ and straight step fluctuations $(\bigcirc)$ is shown as a function of temperature.

tail of this peak extends about to the $m=45$ mode. These two features demonstrate that the detrimental effect of noise is not felt by modes that fall in the spectral window, $5 \leqslant m$ $\leqslant 16$, that is used for the analysis of line tension, and that spatial averaging likewise does not affect mode amplitudes in this spectral window.

Applying this analysis at several temperatures produces the results that are shown in Fig. 4. This figure reveals that the step line tension decreases noticeably with increasing temperature. The data points and error bars in Fig. 4 are the average and the standard deviation, respectively, of the results from typically ten data sets at each temperature. Assuming linear temperature dependence, we find that the line tension varies with a temperature coefficient of $-0.14 \mathrm{meV} / \AA \mathrm{K}$. Repeating the analysis at $1163 \mathrm{~K}$ for islands of different mean radii did not reveal a significant dependence of line tension on radius in the range $1 \mu \mathrm{m}<R$ $<2.1 \mu \mathrm{m}$.

The step line tension is also determined from an evaluation of straight step fluctuations at $1163 \mathrm{~K}$. We analyze the fluctuation behavior of nine steps in four independent image sequences. Each sequence consists of approximately 3000 images, corresponding to a measurement time of $27.5 \mathrm{~min}$. The length of each step that is studied is $L=3400 \mathrm{~nm}$. Additional care is taken to define the mean step position correctly in the straight step geometry. Failure to do so would lead to an artificially low value of stiffness in this or any other analysis that is based on the mean-squared mode amplitude. Of particular concern is the influence of fluctuation modes with wavelengths that are longer than the length $L$ of straight step that is being studied. These modes, which are not analyzed but are necessarily present, push and tilt the mean straight step back and forth on the time scale of their long relaxation time. In order to suppress the influence of the modes that exceed the spatial window of the measurement, a time-varying straight line fit to the rolling time-averaged step shape is used to define the mean step position at every point along the step. A rolling average time frame of 275 s, corresponding to 500 frames, works well without detrimental effects. This choice of rolling average time frame and related technical aspects of the analysis will be discussed further in a forthcoming paper. ${ }^{45}$

The step line tension is determined from straight step fluctuations using Eq. (13) with $L$ replacing $2 \pi R$ and $x_{q}(t)$ 
replacing $r_{q}(t)$ in the denominator. Only the long wavelength modes, $3.6 \times 10^{-4} \AA^{-1} \lesssim q \lesssim 1.1 \times 10^{-3} \AA^{-1}(2 \leqslant m \leqslant 6)$, that have relaxation times exceeding the image integration time and that are sufficiently shorter than the rolling average time frame (see Sec. IV B) are considered. In particular, the $m$ $=2$ mode has a relaxation time that is shorter than $1 / 20$ of the rolling average time frame. The line tension is determined by averaging the values for the temporally resolved modes. The average result and standard deviation for the nine straight steps that were investigated is $\beta$ $=59.5 \pm 4.5 \mathrm{meV} / \AA$. This is only slightly lower than the value that was determined from the analysis of island fluctuations at $1163 \mathrm{~K}$ (Fig. 4), which suggests the consistency of the two methods.

\section{B. Temporal correlations}

We determine mode-dependent relaxation times and obtain information on the rate-limiting kinetics that mediate step motion by evaluating the temporal correlation function of step fluctuations. The mode-dependent time correlation function for straight step fluctuations is defined and can be written in terms of physical quantities as

$$
G_{q}(\Delta t)=\left\langle\left|x_{q}(t+\Delta t)-x_{q}(t)\right|^{2}\right\rangle=A(q)\{1-\exp [-|\Delta t| / \tau(q)]\},
$$

where $\tau(q)$ is the relaxation time, $A(q)=2 k T / L\left(\tilde{\beta} q^{2}+c\right)$, and $c$ is a constant that is related to step repulsions. ${ }^{20,23,40,41,43}$ Replace $x_{q}(t)$ with $r_{q}(t)$ and $L$ with $2 \pi R$ for island fluctuations. For the $\mathrm{Si}(111)(1 \times 1)$ surface, the constant $c$ was shown to be negligible for steps that were more closely spaced than we have investigated here. ${ }^{20}$ The negligible contribution of this constant is confirmed in the analysis of the mode-dependent relaxation time here.

The relaxation time is described by the dynamical scaling relationship $\tau(q)=\tau_{0}(z) q^{-z}$, where different integer values of the dynamical exponent $z$ and correspondingly different forms of $\tau_{0}$ are valid for different dominant kinetic mechanisms. ${ }^{3,4,20,26,35,46}$ For an isolated step, the key mechanisms are identified as periphery diffusion (PD), twodimensional evaporation-condensation (EC) and terrace diffusion (TD). In PD, step motion is mediated by atomic motion along step edges. EC and TD mechanisms both involve exchange of atoms/vacancies between a step and the reservoir on the terrace. The distinction between these two mechanisms is that the step attachment/detachment process is rate limiting in $\mathrm{EC}$, while diffusion is rate limiting in TD. In terms of the kinetic length $d$, discussed in Sec. II, EC corresponds to large kinetic lengths and TD corresponds to small kinetic lengths. The scaling exponents are $z=2,3,4$ for EC, TD, and PD mechanisms, respectively. It should also be noted that a TD behavior is expected to convert to a diffusion-from-step-to-step (DSS) behavior with $z=2$ as step spacing is reduced and steps are no longer isolated. ${ }^{26,35,47}$

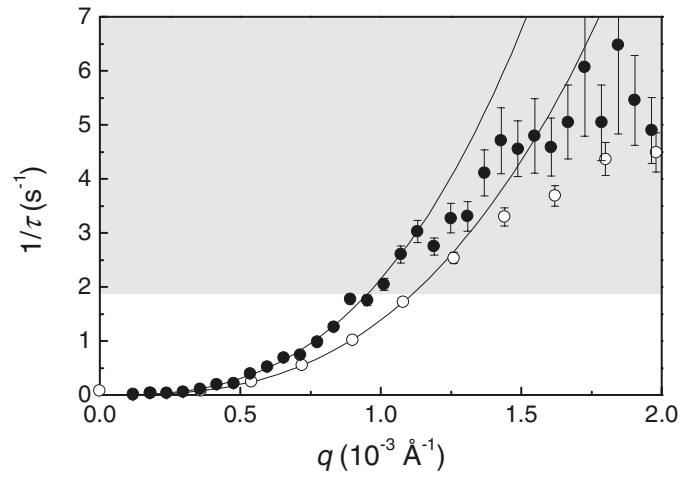

FIG. 5. The dependence of relaxation time upon mode $q$ is shown for island fluctuations $(\bullet)$ and straight step fluctuations $(\bigcirc)$ at $1163 \mathrm{~K}$. The gray shaded area indicates the temporal regime that is shorter than the image integration time. The best fits of the dynamic scaling power law, $[\tau(q)]^{-1}=\tau_{o}^{-1} q^{z}$ that are indicated by the lines through the data are discussed in the text.

The mode-dependent relaxation times that are determined in our investigations for island edge and straight step fluctuations are shown in Fig. 5. This figure first of all demonstrates the point made earlier (see Sec. IV A) that relaxation times simultaneously exceed the image integration time and are significantly shorter than the total measurement time or rolling average time frame for many long wavelength fluctuation modes in both configurations. This confirms that the determination of step stiffness or line tension from these long wavelength modes should be reliable, in principle, from the point of view of the temporal limits of the measurements. Figure 5 also demonstrates another important point made earlier that the investigation of island fluctuations allows for higher $q$ resolution than straight steps for a comparable field of view. We find that relaxation times for the two configurations are comparable. The best fits of the relaxation time scaling law to the data for the temporally resolvable modes are also shown as solid lines in Fig. 5. For island edge fluctuations, the scaling exponent and scale factor that are determined from the average and standard deviation for the ten data sets at this temperature are $z=2.83 \pm 0.10$ and $\left(\tau_{0}\right)^{-1}$ $=(7.80 \pm 6.56) \times 10^{8}$. Similarly, $z=2.74 \pm 0.12$ and $\left(\tau_{0}\right)^{-1}$ $=(3.26 \pm 2.64) \times 10^{8}$ are determined for straight step fluctuations from seven data sets. The results for straight steps and island edges agree within experimental uncertainty. This demonstrates further the consistency between the two methods.

\section{Island decay}

The island decay time was measured as a function of temperature in the range from 1145 to $1380 \mathrm{~K}$. LEEM images that show islands at various stages during decay are presented in Fig. 6 for two temperatures, 1163 and $1283 \mathrm{~K}$. The measured island area vs time is shown in Fig. 7 for three temperatures, 1163,1223 , and $1283 \mathrm{~K}$. This figure also identifies the times $t_{0}$ required for islands to decay from an initial area of $3.46 \mu \mathrm{m}^{2}\left(r_{\mathrm{i}}=1.05 \mu \mathrm{m}\right)$ at the different temperatures. At low temperature, $T \leqq 1200 \mathrm{~K}$, the logarithm of the decay time appears to depend linearly on inverse temperature 


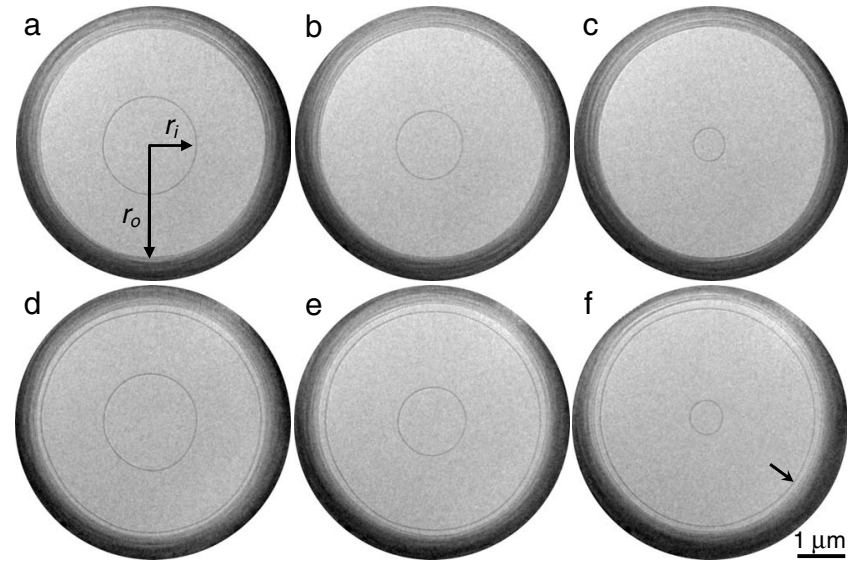

FIG. 6. LEEM images of island decay on the $\mathrm{Si}(111)(1 \times 1)$ surface at $[(\mathrm{a})-(\mathrm{c})] 1163 \mathrm{~K}$ and $[(\mathrm{d})-(\mathrm{f})] 1283 \mathrm{~K}$. The elapsed times after (a) are (b) $341 \mathrm{~s}$ and (c) $527 \mathrm{~s}$, and after (d) are (e) $54 \mathrm{~s}$ and (f) $99 \mathrm{~s}$. The inner $r_{i}$ and outer $r_{o}$ island radii are indicated in (a). In (d)-(f), the outer island also decays slowly, indicated by the arrow in (f), due to desorption from the terrace between the inner and outer island perimeters.

within experimental uncertainty (Fig. 8). According to models of island decay that neglect desorption ${ }^{4-6}$ [see Eq. (11)], this is the expected behavior if the step line tension is assumed to be independent of temperature. A fit of this simplest model to the data below $1200 \mathrm{~K}$ is made by integrating Eq. (11) numerically to determine $t_{0}$, treating the activation energy $E_{1}$ and attempt frequency $\nu_{0}$ as adjustable parameters (dot-dashed curve in Fig. 8). A value of the kinetic length $d=75 a$ is used in the evaluation. This value was determined from a quantitative analysis of island decay and was shown to correspond to the diffusion-limited kinetic regime. ${ }^{33}$ It is also consistent with earlier reports of diffusion-limited step motion during island decay on the $\operatorname{Si}(111)(1 \times 1)$ surface. ${ }^{11,15}$ Assuming that the constant line tension is equal to the value of $66.6 \mathrm{meV} / \AA$, which is determined at $1163 \mathrm{~K}$, this fit yields $E_{1}=1.46 \mathrm{eV}$ and $\nu_{0}=1.35 \times 10^{13} \mathrm{~s}^{-1}$. Different assumed values of constant line tension yield different results for $\nu_{0}$ but do not affect $E_{1}$. However, islands clearly decay faster at $T>1200 \mathrm{~K}$ than indicated by the extrapolation of

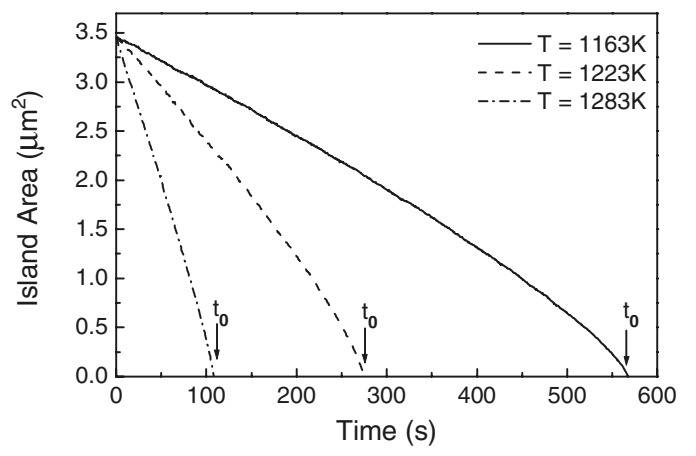

FIG. 7. The island area vs time during decay is shown at three temperatures, $1163 \mathrm{~K}$ (solid line), $1223 \mathrm{~K}$ (dashed line), and $1283 \mathrm{~K}$ (dot-dashed line). The island decay time $t_{0}$ is indicated for each decay temperature. The data were obtained in the symmetric geometry shown in Fig. 6.

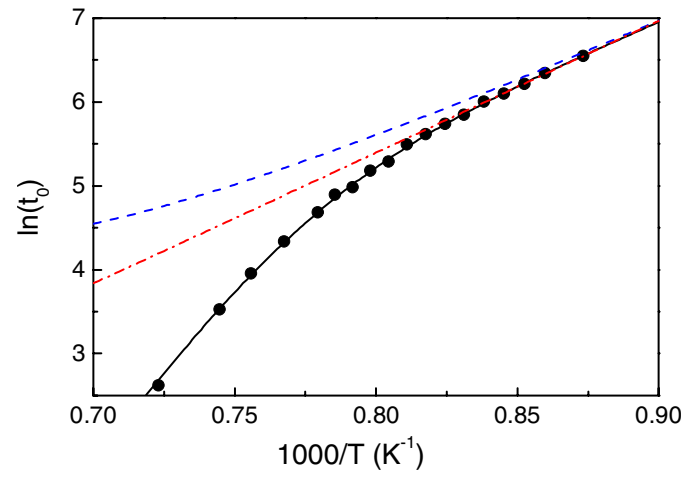

FIG. 8. (Color online) The logarithm of the island decay time (-) is plotted vs inverse temperature for islands of initial area of $3.46 \mu \mathrm{m}^{2}(r=1.05 \mu \mathrm{m})$, corresponding to $t=0$ in Fig. 7. The predictions of the models that include desorption using temperaturedependent line tension (solid curve), neglect desorption using temperature-dependent line tension (dashed curve), and neglect desorption using constant line tension (dot-dashed curve) are shown.

this linear behavior to higher temperature. If the temperaturedependent line tension is now included in the model that neglects desorption, then the predicted linear curve gains some upward inflection with increasing temperature. Consequently, the deviation from the experimental results is even more pronounced. This deviation is due to the effect of desorption. Desorption reduces the adatom concentration on the terrace between the inner and outer island boundaries, including the concentration, $n\left(r_{i}\right)$, at the inner island perimeter. According to Eq. (4), this increases the net detachment current density, which hastens island decay.

The data in Fig. 8 are also fitted with the general model inclusive of desorption (Sec. II), referenced to the temperature-dependent line tension (Fig. 4). The linear dependence of the line tension that is observed between 1145 and $1233 \mathrm{~K}$ is assumed to persist up to the maximum temperature at which island decay was measured. Island decay times are calculated by numerically integrating the sum of Eqs. (10) and (12) with three adjustable parameters, $E_{1}, E_{2}$, and $\nu_{0}$, which affect the decay time. The best fit, indicated by the solid line in Fig. 8, is obtained with $E_{1}=1.53 \mathrm{eV}$, $E_{2}=2.56 \mathrm{eV}$, and $\nu_{0}=2.59 \times 10^{13} \mathrm{~s}^{-1}$. The quality of the fit is excellent. ${ }^{48}$ A comparison is also made in Fig. 8 to the prediction (dashed curve) of the same model using temperaturedependent line tension and the same fit values of $\nu_{0}$ and $E_{1}$, but neglecting desorption $\left(E_{2} \gg 2.56 \mathrm{eV}\right)$. The difference indicates the extent of the role that desorption plays in determining the island decay time.

\section{DISCUSSION}

\section{A. Step line tension}

The analysis of island fluctuations reveals that the step line tension decreases between 1145 and $1233 \mathrm{~K}$ with a linear temperature coefficient of $-0.14 \mathrm{meV} / \AA \mathrm{K}$. The reasonable agreement between the results obtained here from the analysis of straight step and island fluctuations at $1163 \mathrm{~K}$ also suggests that the magnitude of the line tension is deter- 
mined correctly. For comparison, the two most recent results that were derived from straight step fluctuations that were observed using REM, $46 \mathrm{meV} / \AA$ at $1173 \mathrm{~K}$ (Ref. 19) and between 22.8 and $31.9 \mathrm{meV} / \AA$ at $1373 \mathrm{~K}$ (Ref. 24), indicate a decrease of $30 \%-50 \%$ over this $200 \mathrm{~K}$ range. A similar decrease of about $40 \%$ is determined over the same temperature range by extrapolating our results, with the assumption that the temperature dependence remains linear above $1233 \mathrm{~K}$. This is comparable to an approximate $35 \%$ decrease that was predicted in the range of $1173-1373 \mathrm{~K}$ by statistical mechanical model calculations. ${ }^{16}$ However, there is still clearly a significant disagreement between the magnitudes of our results and those reported earlier. ${ }^{19,24}$ Our result is about $40 \%$ higher.

One possible explanation for this discrepancy is that there may be an error in the absolute temperature measurement in our experiment or the earlier set of experiments. The similarity of the temperature dependence of the stiffness is a fair indication that the relative temperature measurement is comparable in the two cases. We measured temperature using a thermocouple and an optical pyrometer that were calibrated carefully against the $(7 \times 7)$ phase transition temperature, which could be easily identified with LEEM. This calibration was checked in the imaged sample area before and after each measurement and was very reproducible. The acceptance of the absolute temperature scale in our experiments necessarily implies a $150 \mathrm{~K}$ error in the REM experiments, which seems unlikely.

An alternative explanation of the discrepancy is that it originates in details of the analysis that were mentioned in Sec. IV A. In particular, the implementation of the straight step analysis demands that the mean step position along the step be defined correctly. Any misjudgment of the mean step position will lead to an overestimation of fluctuation amplitudes, $\left|x_{q}(t)\right|^{2}$, and, consequently, to an underestimation of stiffness or line tension. Although it may be convenient to use a single straight line fit to the (total measurement) timeaveraged step shape to define the mean step position at every point along the step, this neglects the influence of fluctuation modes with wavelengths that are longer than the length $L$ of step that is being analyzed. These ultralong wavelength modes were approximately taken into account in our analysis (Sec. IV A) by using a time-varying straight line fit to the rolling time-averaged step shape to define the mean step position. The time-varying straight line mainly rotates with varying azimuthal angle with respect to the total timeaveraged straight line fit to the step. This approach produces a result for line tension that is in good agreement with the result that is obtained from the analysis of island edge fluctuations. If the rolling average definition of the mean step position is not used, then a line tension about half as large would be determined in the present case. On the contrary, the evaluation of island edge fluctuations is not susceptible to this systematic error because there can be no modes with wavelengths longer than the perimeter of the island. The technical aspects of our analysis will be discussed further in a forthcoming paper. ${ }^{45}$ To the best of our knowledge, the time-varying definition of the mean step position was not used in the prior investigations of straight step fluctuations on the $\operatorname{Si}(111)$ surface. ${ }^{17-21,23,24}$

\section{B. Temporal correlations}

The temporal correlations of step fluctuations at island edges and of straight steps consistently show here that the inverse of the relaxation time scales nearly with the cube of the mode, $q$. This is notably different from the quadratic dependence that was determined earlier for straight step fluctuations with REM. ${ }^{20,23}$ A quadratic dependence may be indicative of either an EC or DSS mechanism of step motion. ${ }^{3,20,26,35}$ Note that the EC mechanism identifies the step attachment/detachment process as rate limiting, which implies a large kinetic length. This interpretation clearly contradicts the diffusion-limited behavior and small kinetic length, $d \sim 75 a$, that were determined from observations of island decay behavior. ${ }^{11,15,33}$ Rather, the diffusion-limited (small $d$ ) process, manifests itself in the TD mechanism of step motion, with its signature cubic dependence of the inverse relaxation time upon $q$. Fluctuation dynamics have usually been attributed to the EC mechanism in the past, and evidence of TD behavior in temporal correlations is very rare. The only previous reports of TD behavior in fluctuation dynamics in a physical system are for the $\mathrm{Pt}(111)$ and $\operatorname{Pd}(111)$ surfaces $^{43}$ and for $\mathrm{Cu}(111)$ electrodes in an electrolyte. ${ }^{49}$

As noted before, a transition from a TD to a DSS behavior with decreasing step spacing can produce a crossover from cubic to quadratic dependence of the inverse relaxation time. Such a transition may explain the discrepancy between the cubic scaling that is indicated here and the quadratic scaling that was observed earlier. ${ }^{20,23}$ In particular, the step spacing in the earlier investigations was $L_{s} \sim 0.15-0.25 \mu \mathrm{m}^{20,23}$ This is considerably smaller than the average spacing between the nearest steps here of $L_{s}=0.65 \mu \mathrm{m}$ for island fluctuations and $L_{s} \sim 0.50 \mu \mathrm{m}$ for straight step fluctuations. Clearly, further investigations are needed to test this explanation. An extension of the current investigation to examine fluctuations of different island sizes on fixed platforms may be a fruitful way to carry out this test. Note that one can also distinguish EC from DSS by a careful examination of step correlations, as described in Ref. 50. In that case, steps on the $\mathrm{Si}(111)-\sqrt{3} \times \sqrt{3} R 30^{\circ} \mathrm{Al}$ surface at $970 \mathrm{~K}$ were shown to have an EC rather than a DSS behavior. Alternatively, the discrepancy on the scaling exponent may be related to the method of sample heating. The value of $z=2$ was obtained earlier using direct current heating, which is known to cause electromigration. Electromigration is known to induce numerous step morphological phenomena. ${ }^{3,14,31}$ The sample heating in our experiments was performed using electron bombardment, which does not induce electromigration.

Until this issue can be resolved, we interpret our results to mean that the intrinsic mechanism of isolated step motion on the $\operatorname{Si}(111)(1 \times 1)$ surface is TD. Using the TD scaling form, ${ }^{3,42} \tau_{q}=k T / \widetilde{\beta} D_{s} n_{e q} \Omega^{2} q^{3}$, to interpret the scaling factors determined experimentally at $1163 \mathrm{~K}\left[\left(\tau_{0}\right)^{-1}=(7.8 \pm 6.6)\right.$ $\times 10^{8}$ for island fluctuations and $\left(\tau_{0}\right)^{-1}=(3.3 \pm 2.6) \times 10^{8}$ for straight steps], we obtain $D_{s} n_{e q}=(6.2 \pm 5.2) \times 10^{6} \mathrm{~s}^{-1}$ and $(2.6 \pm 2.1) \times 10^{6} \mathrm{~s}^{-1}$, respectively. These scaling factors are obtained for the best-fit dynamical scaling exponents of $z$ $=2.74$ and $z=2.83$ for islands and straight steps, respectively, 
instead of $z=3$ indicated in the scaling form. Larger values, $\left(\tau_{0}\right)^{-1}=(2.2 \pm 0.3) \times 10^{9} \mathrm{~s}^{-1}$ for islands and $\left(\tau_{0}\right)^{-1}$ $=(1.6 \pm 0.1) \times 10^{9} \mathrm{~s}^{-1}$ for straight steps, are obtained by fitting temporal correlations with the dynamic exponent constrained to be $z=3$. The quality of the fit curves is also very good in this case. These lead to $D_{s} n_{e q}=(1.8 \pm 0.4) \times 10^{7} \mathrm{~s}^{-1}$ and $(1.3 \pm 0.1) \times 10^{7} \mathrm{~s}^{-1}$, respectively, for islands and straight steps. For comparison, a value of $D_{s} n_{e q}$ $=\left(\frac{3}{2} a^{2} \nu_{0} e^{-E_{d i f} / k T}\right)\left(\Omega^{-1} e^{-E_{a d} / k T}\right)=\sqrt{3} \nu_{0} e^{-E_{1} / k T}=2.11 \times 10^{7} \mathrm{~s}^{-1}$ is obtained using $\nu_{0}=2.59 \times 10^{13} \mathrm{~s}^{-1}$ and $E_{1}=E_{a d}+E_{\text {dif }}$ $=1.53 \mathrm{eV}$, which were determined from the analysis of island decay in Sec. IV C. This value of $D_{s} n_{e q}$ is in better and reasonable agreement with the values determined from temporal correlations with dynamic scaling exponent $z=3$. The $D_{s} n_{e q}$ that was determined earlier from LEEM observations of island decay is about $1.8 \times 10^{7} \mathrm{~s}^{-1}$ at $1163 \mathrm{~K},{ }^{11}$ or double that if the correct value of the stiffness would have been used in the evaluation. A value of $D_{s} n_{\mathrm{eq}}=1 \times 10^{8} \mathrm{~s}^{-1}$ was obtained from REM data using an approximate formula appropriate for a DSS mediated mechanism of step motion. ${ }^{26}$ Similarly, if we reinterpret an earlier temporal correlation behavior ${ }^{20}$ in terms of a TD mechanism, then we obtain a value of $D_{s} n_{e q}$ $=2.1 \times 10^{8} \mathrm{~s}^{-1}$, which is larger by a factor of about 10 than the result obtained in our analysis. Another value that was determined at $1373 \mathrm{~K}$ by treating temporal correlations under electromigration conditions in the context of a DSS mechanism yielded $D_{s} n_{e q} \approx 2 \times 10^{11} \mathrm{~s}^{-1}$, which was noted to be particularly high. ${ }^{23}$ This cannot be accounted for by extrapolating the present and earlier ${ }^{11}$ results obtained with LEEM at $1163 \mathrm{~K}$ to higher temperature.

\section{Island decay}

The value of the activation energy $E_{1}=E_{a d}+E_{d i f}$ $=1.53 \mathrm{eV}$ that is determined from island decay here is in the middle of the range of values that were determined previously, $1.1 \mathrm{eV},{ }^{13} 1.9 \mathrm{eV},{ }^{27}$ and $1.3 \mathrm{eV} .{ }^{11}$ The value of the activation energy, $E_{2}=E_{d e s}-E_{d i f}=2.56 \mathrm{eV}$, that we determine is also just a little larger than the result that was obtained earlier, $2.4 \mathrm{eV} .{ }^{27}$ Our result for the sum $E_{1}+E_{2}=E_{a d}+E_{d e s}$ $=4.09 \mathrm{eV}$, which represents the sublimation energy, is also in the vicinity of the earlier reported values of the sublimation energy, $4.3 \mathrm{eV}$ (Ref. 27) and $4 \mathrm{eV}$ (Ref. 18), which were determined by other methods. The attempt frequency that we determine, $\nu_{0}=2.59 \times 10^{13} \mathrm{~s}^{-1}$, is also physically reasonable. We take the adatom pair formation energy to be $E_{a d}$ $=0.23 \mathrm{eV}$. This value of $E_{\text {ad }}$ produces an equilibrium concentration near a straight step, $n_{\mathrm{eq}}(\infty)$, that is consistent with the equilibrium coverage of $\sim 0.20-0.22 \mathrm{ML}$ at $1173 \mathrm{~K}$ that was reported earlier. ${ }^{25}$ Then, the diffusion energy, $E_{d i f}=1.30 \mathrm{eV}$, and the desorption energy, $E_{d e s}=3.86 \mathrm{eV}$, are determined from $E_{1}$ and $E_{2}$.

It is instructive at this point to examine the concentration profile that is predicted by Eqs. (7), (9a), and (9b). Figure 9 shows the predicted profiles between the inner and outer island boundaries at 1163 and $1283 \mathrm{~K}$. The inner, $r_{i}$ $=1.05 \mu \mathrm{m}$, and outer, $r_{0}=2.35 \mu \mathrm{m}$, island radii in this figure correspond to the island decay geometry that was investigated experimentally (Fig. 6). The concentration profiles
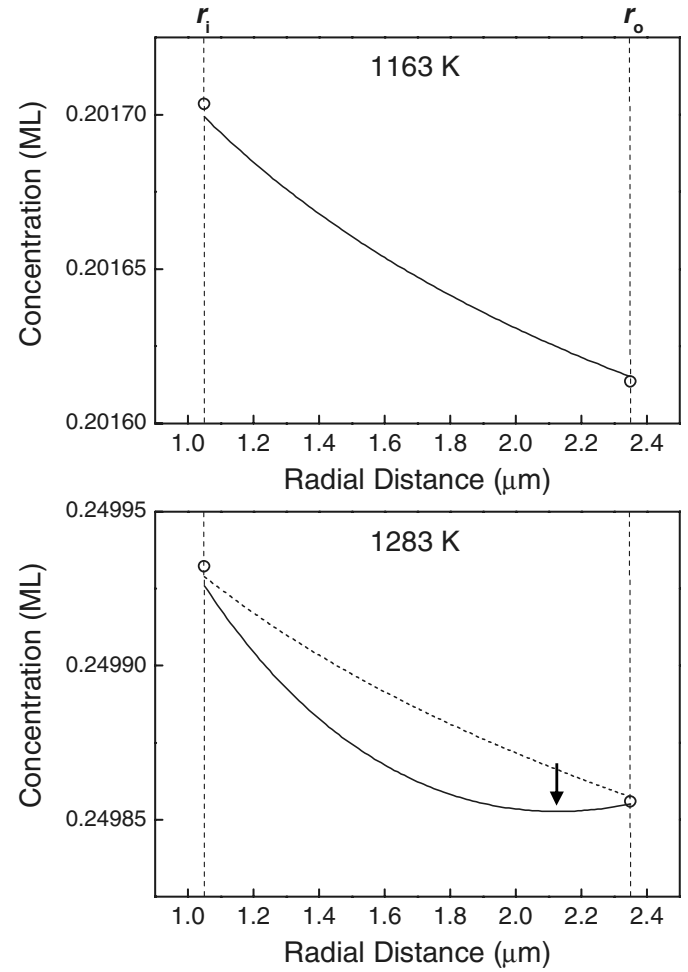

FIG. 9. The radial dependence of the adatom concentration given by Eqs. (7), (9a), and (9b) is shown between the inner $r_{i}$ and outer $r_{o}$ island radii at $1163 \mathrm{~K}$ (upper) and $1283 \mathrm{~K}$ (lower). The open circles indicate the equilibrium concentrations given by Eq. (2), and the arrow identifies a minimum that is produced by desorption. The solid curves represent the concentrations in the initial island configurations at $1163 \mathrm{~K}$ in Fig. 6(a) and at $1283 \mathrm{~K}$ in Fig. $6(\mathrm{~d})$. The dotted curve at $1283 \mathrm{~K}$ indicates the concentration profile if desorption was to be neglected at this temperature.

(solid curves in Fig. 9) are produced using the parameters that were determined in Sec. IV C, $E_{1}=1.53 \mathrm{eV}, E_{2}$ $=2.56 \mathrm{eV}$, and $\nu_{0}=2.59 \times 10^{13} \mathrm{~s}^{-1}$. The value of the line tension that is used to generate the profile at $1163 \mathrm{~K}, \beta$ $=66.6 \mathrm{meV} / \AA$, was determined directly from island edge fluctuations at this temperature (Sec. IV A). The value at $1283 \mathrm{~K}, 50.3 \mathrm{meV} / \AA$, is obtained by extrapolating the results of Sec. IV A to this higher temperature. An adatom pair formation energy, $E_{a d}=0.23 \mathrm{eV}$, and a kinetic length of $d$ $=75 a$ are also used to produce the profiles in Fig. 9. The kinetic length was determined previously at $1163 \mathrm{~K}$ (Ref. 33 ) and should not change significantly in the narrow temperature range that we are considering.

Figure 9 first of all shows that a higher concentration is present on the surface at higher temperature, consistent with Eq. (2). At low temperature, illustrated by the profile at $1163 \mathrm{~K}$ in Fig. 9, the concentration has the usual form that mediates island decay. In particular, the actual concentrations at the inner and outer island perimeters are, respectively, lower and higher than the equilibrium concentrations, indicated by open circles in the figure. This is consistent with the concentration gradients at the boundaries through the boundary conditions in Eqs. (8a) and (8b). The concentration profile (Fig. 9) and the decay time (Fig. 8) at this temperature are little affected by desorption. We expect that the outer 
island radius should increase during the decay of the inner island due to mass accumulation. However, this does not occur because of the constraint imposed by the mound geometry in our experiments [Figs. 6(a)-6(c)]. Atoms that reach the outer island perimeter must therefore escape by descending the mound sideface through a series of attachment and detachment processes at successively lower levels.

At suitably high temperature, illustrated by the profile at $1283 \mathrm{~K}$ in Fig. 9, desorption produces a minimum in the concentration profile at a position between the inner and outer island edges, indicated by the arrow in the figure. The actual concentration is also lower than the equilibrium concentration at the outer perimeter, $n\left(r_{0}\right)<n_{e q}\left(r_{0}\right)$, at this temperature. This boundary concentration relation is consistent with the concentration gradient at the outer perimeter through the boundary condition [Eq. (8b)]. The physical meaning of this is that there is now also a net detachment from the outer island perimeter and radially inward transport of adatoms. This implies that the outer island must also decay. This prediction is confirmed by the experimental observations at $1283 \mathrm{~K}$, shown in Figs. 6(d)-6(f), that the outer island decays slowly. The model also predicts that the concentration profile has a slope of zero and that the concentration is equal to the equilibrium value at the outer island perimeter at $1263 \mathrm{~K}$. This coincides with the experimental observation that outer island decay of the sort that is depicted in Figs. 6(d)-6(f) is only observed at $T \geqslant 1265 \mathrm{~K}$.

The model that is discussed in this paper considers that mass transport during island decay is mediated by diffusion of adatoms that are produced at steps. However, it was shown ${ }^{51}$ that adatom-vacancy pair formation plays a dominant role in mass transport during the $(7 \times 7)-(1 \times 1)$ phase transition. In particular, $(7 \times 7)$ domains are converted to $(1 \times 1)$ structure when adatoms that are created (together with vacancies) on $(1 \times 1)$ regions of terraces migrate to $(7$ $\times 7)$ domain edges. At the same time, the vacancies that are left behind migrate to steps where they are annihilated. This adatom-vacancy mechanism replaces direct communication between adatom formation at steps and adatom absorption at $(7 \times 7)$ domain edges. A crucial element of the model used to describe the $(7 \times 7)$ domain decay kinetics is that the adatom and vacancy concentrations are far below their equilibrium values during the phase transition. ${ }^{51}$ This means that adatom formation at steps must be suppressed for some reason. This condition is very likely caused by the continuous decoration of steps by $(7 \times 7)$ structure during the phase transition. In the absence of the step adatom source, creation of adatomvacancy pairs on terraces becomes important, despite the high pair formation energy, $3.6-3.8 \mathrm{eV},{ }^{51}$ which is very close to the adatom desorption energy determined here.

Adatom-vacancy formation on terraces should not be important during island decay for several reasons. First of all, steps are not decorated with a $(7 \times 7)$ structure and the adatom concentration on terraces is also close to equilibrium during island decay. According to the $(7 \times 7)$ domain decay kinetics model in Ref. 51, this strongly diminishes the importance of adatom-vacancy pair formation in mass transport. Second, fast diffusion and slow adatom detachment from steps is implicit in the domain decay kinetics model. This corresponds to the attachment-detachment limited kinetic regime. On the contrary, there is considerable experimental evidence ${ }^{11,33}$ that step motion during island decay on the $\mathrm{Si}(111) \quad(1 \times 1)$ surface is diffusion limited, not attachment-detachment limited. The contrast between domain and island decay kinetics is a sign that the dominant kinetic processes are fundamentally different.

\section{CONCLUSIONS}

We have determined the step line tension on the $\mathrm{Si}(111)$ $(1 \times 1)$ surface by a capillary wave analysis of step fluctuations that were observed with LEEM. Our investigations of the step line tension are probably the most comprehensive to date on the $\mathrm{Si}(111)(1 \times 1)$ surface. Notable features of the present work in this regard include (a) the comparison of fluctuations in two configurations (island edges and straight steps), (b) measurements at multiple temperatures in series on the same sample, and (c) careful averaging of results that are obtained from the analysis of multiple $(\sim 10)$ independent data sets at each temperature. These attributes contribute to the reliability of the magnitude and temperature dependence of the step line tension that are reported here. This is a compelling reason to adopt the present results in the analysis and modeling of step morphological evolution on the $\mathrm{Si}(111)(1 \times 1)$ surface in the future. In the course of these investigations, temporal correlations of step fluctuations were found to exhibit the signature of a terrace diffusion-limited mechanism of step motion. The importance of these results is demonstrated immediately here by measurements of island decay on the $\operatorname{Si}(111)(1 \times 1)$ surface. Evaluation of the island decay time with a general model of island decay inclusive of desorption and referenced to the temperature-dependent line tension accurately determines activation energies that are relevant to mass transport and sublimation.

\section{ACKNOWLEDGMENTS}

Work at HKUST was supported by the Hong Kong Research Grants Council under Grant No. HKUST600103. Work at the University of Maryland was supported primarily by UMD NSF-MRSEC under Grant No. DMR 05-20471, with partial support from DOE CMSN Grant No. DEFG0205ER46227. 
*Corresponding author: phaltman@ust.hk

†Present address: Laboratory of Receptor Biology and Gene Expression, NCI, National Institutes of Health, Bethesda, Maryland 20893, USA.

${ }^{1}$ P. Nozières, in Solids Far From Equilibrium, edited by C. Godrèche (Cambridge University Press, Cambridge, England, 1991), p. 1.

${ }^{2}$ L. D. Landau and E. M. Lifshitz, Statistical Physics (AddisonWesley, Reading, MA, 1969), Chap. 15.

${ }^{3}$ H. C. Jeong and E. D. Williams, Surf. Sci. Rep. 34, 171 (1999).

${ }^{4}$ M. Giesen, Prog. Surf. Sci. 68, 1 (2001).

${ }^{5}$ P. Wynblatt and N. A. Gjostein, in Progress in Solid State Chemistry, edited by J. O. McCardin and G. Somorjai (Pergamon, Oxford, 1975), Vol. 9, p. 21.

${ }^{6}$ J. G. McLean, B. Krishnamachari, D. R. Peale, E. Chason, J. P. Sethna, and B. H. Cooper, Phys. Rev. B 55, 1811 (1997).

${ }^{7}$ B. Krishnamachari, J. McLean, B. Cooper, and J. Sethna, Phys. Rev. B 54, 8899 (1996).

${ }^{8}$ A. V. Latyshev, H. Minoda, Y. Tanishiro, and K. Yagi, Phys. Rev. Lett. 76, 94 (1996).

${ }^{9}$ G. S. Bales and A. Zangwill, Phys. Rev. B 41, 5500 (1990).

${ }^{10}$ G. Schulze Icking-Konert, M. Giesen, and H. Ibach, Surf. Sci. 398, 37 (1998).

${ }^{11}$ H. Hibino, C. W. Hu, T. Ogino, and I. S. T. Tsong, Phys. Rev. B 63, 245402 (2001).

${ }^{12}$ K. Morgenstern, Phys. Status Solidi B 242, 773 (2005).

${ }^{13}$ A. Pimpinelli, I. Elkinani, A. Karma, C. Misbah, and J. Villain, J. Phys.: Condens. Matter 6, 2661 (1994).

${ }^{14}$ O. Pierre-Louis, C. R. Phys. 6, 11 (2005).

${ }^{15}$ K. L. Man, W. X. Tang, X. F. Jin, and M. S. Altman, Surf. Interface Anal. 38, 1632 (2006).

${ }^{16}$ N. Akutsu and Y. Akutsu, J. Phys.: Condens. Matter 11, 6635 (1999).

${ }^{17}$ C. Alfonso, J. M. Bermond, J. C. Heyraud, and J. J. Métois, Surf. Sci. 262, 371 (1992).

${ }^{18}$ J. M. Bermond, J. J. Métois, J. C. Heyraud, and C. Alfonso, Surf. Sci. 331-333, 855 (1995).

${ }^{19}$ J. M. Bermond, J. J. Métois, J. C. Heyraud, and F. Floret, Surf. Sci. 416, 430 (1998).

${ }^{20}$ N. C. Bartelt, J. L. Goldberg, T. L. Einstein, E. D. Williams, J. C. Heyraud, and J. J. Métois, Phys. Rev. B 48, 15453 (1993).

${ }^{21}$ T. Suzuki, J. J. Métois, and K. Yagi, Surf. Sci. 339, 105 (1995).

${ }^{22}$ J. M. Bermond, J. J. Métois, X. Egéa, and F. Floret, Surf. Sci. 330, 48 (1995).

${ }^{23}$ S. D. Cohen, R. D. Schroll, T. L. Einstein, J. J. Métois, H. Gebremariam, H. L. Richards, and E. D. Williams, Phys. Rev. B 66, 115310 (2002).

${ }^{24}$ J. J. Métois and P. Müller, Surf. Sci. 548, 13 (2004).

${ }^{25}$ Y. N. Yang and E. D. Williams, Phys. Rev. Lett. 72, 1862 (1994).
${ }^{26}$ A. Pimpinelli, J. Villain, D. E. Wolf, J. J. Métois, J. C. Heyraud, I. Elkinani, and G. Uimin, Surf. Sci. 295, 143 (1993).

${ }^{27}$ Y. Homma, H. Hibino, T. Ogino, and N. Aizawa, Phys. Rev. B 58, 13146 (1998).

${ }^{28}$ A. Saul, J. J. Metois, and A. Ranguis, Phys. Rev. B 65, 075409 (2002).

${ }^{29}$ A. V. Latyshev, A. B. Krasilnikov, A. L. Aseev, L. V. Sokolov, and S. I. Stenin, Surf. Sci. 254, 90 (1991).

${ }^{30}$ T. Zhao, J. D. Weeks, and D. Kandel, Phys. Rev. B 70, 161303(R) (2004).

${ }^{31}$ K. Yagi, H. Minoda, and M. Degawa, Surf. Sci. Rep. 43, 45 (2001).

${ }^{32}$ Y. Homma and P. Finnie, J. Phys.: Condens. Matter 11, 9879 (1999).

${ }^{33}$ K. L. Man, A. B. Pang, and M. S. Altman, Surf. Sci. 601, 4669 (2007).

${ }^{34}$ F. Szalma, H. Gebremariam, and T. L. Einstein, Phys. Rev. B 71, 035422 (2005).

${ }^{35}$ S. V. Khare and T. L. Einstein, Phys. Rev. B 57, 4782 (1998).

${ }^{36}$ T. Ala-Nissila, R. Ferrando, and S. C. Ying, Adv. Phys. 51, 949 (2002).

${ }^{37}$ S. C. Jain, S. K. Agarwal, W. N. Borle, and S. Tata, J. Phys. D 4, 1207 (1971).

${ }^{38}$ E. Bauer, Rep. Prog. Phys. 57, 895 (1994).

${ }^{39}$ W. F. Chung and M. S. Altman, Ultramicroscopy 74, 237 (1998).

${ }^{40}$ N. C. Bartelt, T. L. Einstein, and E. D. Williams, Surf. Sci. Lett. 240, L591 (1990).

${ }^{41}$ N. C. Bartelt, R. M. Tromp, and E. D. Williams, Phys. Rev. Lett. 73, 1656 (1994).

${ }^{42}$ N. C. Bartelt, T. L. Einstein, and E. D. Williams, Surf. Sci. 312, 411 (1994).

${ }^{43}$ M. Ondrejcek, W. Swiech, M. Rajappan, and C. P. Flynn, Phys. Rev. B 72, 085422 (2005).

${ }^{44}$ C. Tao, T. J. Stasevich, T. L. Einstein, and E. D. Williams, Phys. Rev. B 73, 125436 (2006).

${ }^{45}$ K. L. Man, F. Szalma, T. L. Einstein, and M. S. Altman (unpublished).

${ }^{46}$ M. Bisani and W. Selke, Surf. Sci. 437, 137 (1999).

${ }^{47}$ B. Blagojević and P. M. Duxbury, Phys. Rev. E 60, 1279 (1999).

${ }^{48}$ Increasing the kinetic length to $d=3000 a$ in the attachmentdetachment limited regime does not affect $E_{1}$, produces an increase in $E_{2}$ of less than $0.1 \mathrm{eV}$, and mainly increases $\nu_{0}$ by a factor of about 3 .

${ }^{49}$ S. Baier, S. Dieluweit, and M. Giesen, Surf. Sci. 502-503, 463 (2002).

${ }^{50}$ D. B. Dougherty, I. Lyubinetsky, T. L. Einstein, and E. D. Williams, Phys. Rev. B 70, 235422 (2004).

${ }^{51}$ J. B. Hannon, H. Hibino, N. C. Bartelt, B. S. Swartzentruber, T. Ogino, and G. Kellogg, Nature (London) 405, 552 (2000). 